\title{
Medical Students' Studying Approach to a Changing Medical School Anatomy Curriculum
}

\author{
Daniel Gibson, BA ${ }^{1}$, Polly Husmann, $\mathrm{PhD}^{2}$ \\ ${ }^{1}$ Indiana University School of Medicine, ${ }^{2}$ Medical Sciences Program, Indiana \\ University School of Medicine
}

\section{Background and Hypothesis:}

With medical school curriculum changes, students have to reassess how they approach their studies. This study evaluates how medical students planned to study gross anatomy before and after the 2016 Indiana University School of Medicine (IUSM) curriculum change. Student study plans are hypothesized to be different before and after the curriculum change.

\section{Methods:}

First-year medical students from 2015-2017 were given a survey prior to their gross anatomy course. The survey integrated 37 Likert scale questions about resources the students might use, study strategies, demographics, prior anatomy and/or physiology courses, etc. T-tests were run between the curriculum and student grades as well as seven categories that grouped related questions from the survey. Comparisons were also made between the upper and lower thirds of the class.

\section{Results:}

The final course and NMBE percentages were found to be significantly different between curricula $(p<0.0001 / p<0.0001)$. Differences in planned usage of textbased, lab-based, and web-based resources were also significant $(p<0.0001 / p=0.002 / p=0.007)$. Analyses additionally found that students in the upper third of all classes were more likely to have a prior anatomy and physiology course $(p=0.031)$.

\section{Conclusion and Potential Impact:}

Student study plans for anatomy have changed with the revision of the curriculum. Current students are planning to use outside resources (e.g., textbooks, websites) more than previous cohorts in the traditional curriculum. In addition, students with prior knowledge of anatomy and physiology may have an advantage in understanding medical anatomy. These findings have implications for the long-term retention of this material and subsequent student outcomes. 